\title{
Reply to comment on "Impact and cost-effectiveness of a universal strategy to promote physical activity in primary care"
}

\author{
Martin Gulliford · Judith Charlton • \\ Nawaraj Bhattarai · Caroline Rudisill
}

Published online: 10 March 2015

(c) Springer-Verlag Berlin Heidelberg 2015

\section{Dear Editor,}

Thank you for the opportunity to reply to the reader's comment. The comment appears to mis-understand two aspects of our study.

Firstly, our study did not address the general question of whether physical activity is beneficial for health. A considerable body of evidence suggests this is the case. Rather, our study considered whether one particular intervention, deployed in a particular population with the aim of increasing physical activity, might prove cost-effective. It is entirely possible that some interventions to promote physical activity may not be cost-effective.

Secondly, the comment appears to have overlooked the probabilistic nature of our model, which incorporated estimates of random error for all data inputs. Random error arises not only from imprecision in the measure of intervention effect, but also from the use of estimates for disease incidence and mortality that were estimated from empirical data sources and incorporated into the model. Probabilistic simulation did not entirely exclude the possibility that life years might be lower in intervention than comparator scenarios. However, the estimate of $29 \%$ for the probability that life years were less after intervention, represents a considerable "improvement" on the $50 \%$ value that would be expected in the event that a brief lifestyle intervention had a similar effect on physical activity as usual care. This estimate therefore draws attention to the very small and uncertain effect size associated with brief primary care interventions to promote physical activity, and their limited cost-effectiveness when employed in a universal intervention strategy.

Finally, we note that it is well recognized that initiation of physical activity may be associated with cardiovascular events and sudden cardiac death [1], making it important to assess possible harms as well as benefits of interventions.

\section{Reference}

1. Thompson, P.D., Franklin, B.A., Balady, G.J., Blair, S.N., Corrado, D., Estes 3rd, N.A., et al.: Exercise and acute cardiovascular events placing the risks into perspective: a scientific statement from the American Heart Association Council on Nutrition, Physical Activity, and Metabolism and the Council on Clinical Cardiology. Circulation 115, 2358-2368 (2007)

This reply refers to the article available at doi:10.1007/s10198-015-0674-0.

M. Gulliford $(\bowtie) \cdot$ J. Charlton · N. Bhattarai · C. Rudisill Department of Primary Care and Public Health Sciences, King's College London, London, UK e-mail: martin.gulliford@kcl.ac.uk

M. Gulliford · J. Charlton · N. Bhattarai · C. Rudisill Department of Social Policy, London School of Economics, London, UK 